\title{
Long Term Effects of Radiation-Induced Osteonecrosis of the Pelvis Caused Complete Failure of Total Hip Arthroplasty
}

\author{
Tigist Wodaje, Per Wretenberg \\ Department of Molecular Medicine and Surgery, Section of Orthopaedics, Karolinska Institute \\ and Karolinska University Hospital, Stockholm, Sweden \\ Email: per.wretenberg@karolinska.se
}

Received May 25, 2011; revised May 21, 2012; accepted June 10, 2012

\begin{abstract}
This case report describe severe osteonecrosis of the pelvis more than 30 years after radiation due to ovarian cancer. As orthopedic surgeon it is important to ask about previous radiation to the pelvis in case of total hip arthroplasty and also to perform CT in unclear cases.
\end{abstract}

Keywords: Osteonecrosis; Hip; Radiation

\section{Case Report}

A woman born in 1936 was operated in 1976 for ovarian cancer. After adjuvant cytostatic chemotherapy and radiotherapy of $40 \mathrm{~Gy}$ to the lower abdominal field she was free of cancer. In 2007 she was operated for spinal stenosis with laminectomy and fusion. Total hip replacement (THR) due to osteoarthrosis in the right hip was done successfully 2008. In October 2009 she was diagnosed with highly malignant B-cell lymphoma and was treated with cytostatic chemotherapy followed by radiotherapy 40 Gy to the left supracalavicular fossa.

In July 2010 she was diagnosed with coxarthrosis of the left hip and put on waiting list for THR. Two weeks later the patient arrived to the emergency department with severe pain and walking problems. X-ray showed destruction of left caput femoris, Figure 1. She was operated as emergency case on 09-08-10 with cemented THR and reinforcement ring, Figure 2.

After the left side THR the patient had good functional progress the first two months. She could walk without pain using rolling walker and return to home. After two months she felt sudden pain in the left hip and X-ray showed complete loosening of the acetabular implant Figure 3.

11-10-2010 she was reoperated with the aim to reimplant an acetabular component. During operation neither tumor nor infection could be ruled out and therefore several cultures and biopsies were taken and the acetabular component was removed. The femoral component was left in place and she was left with a modified Girdelstone situation. All samples were negative for infection and PAD was negative for tumor. CT showed irregular sclerotic destruction likely from radiotherapy in almost all parts of os illium, os ischium and ramus superior. 07-122010 a reconstruction was done with a METS Cone hemipelvic prosthesis (Stanmore, UK) and a cemented cup Figure 4.

About ten days after the secondary hemipelvic prosthesis the patient had clear signs of infection with fever and a high C-Reactive-Protein (CRP). She was put on intravenous Tazocin $^{\odot}$ (piperacillin with tazobactam) $4 \mathrm{~g}$ $\times 3$. The wound started oozing large amounts of serous fluid and surgical revision of the wound was done 23-122010. All samples showed growth of enterococcus faecalis and the patient had good coverage with Tazocin. However, a month later the patient's general condition

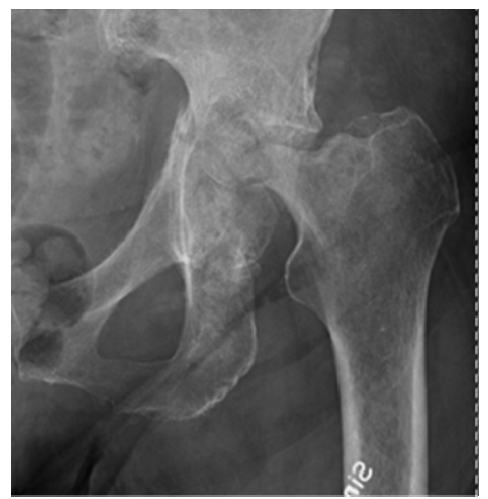

Figure 1. Severe rapid destruction of the caput femoris and acetabular fracture. 


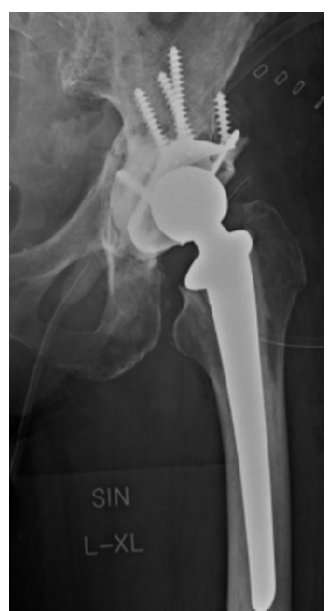

Figure 2. The left hip after reconstruction with reinforcement ring and cemented THR.

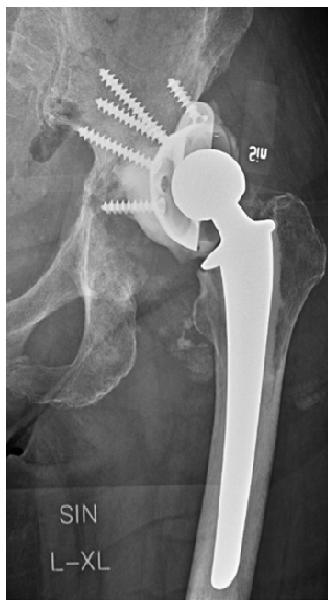

Figure 3. Complete loosening of the acetabular reconstruction.

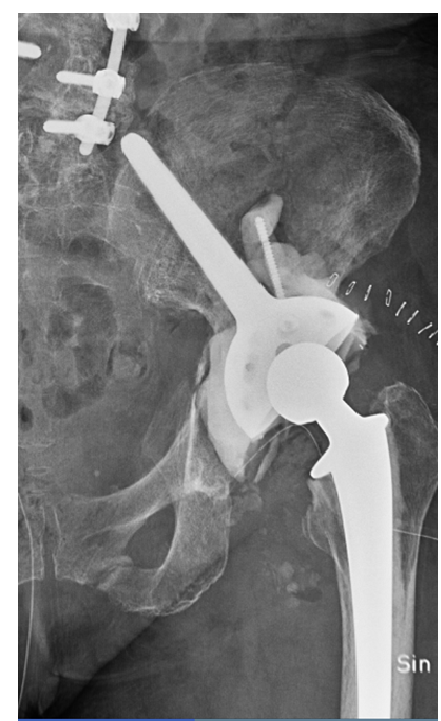

Figure 4. Reconstruction with METZ hemipelvis cone prosthesis. and infection parameters could not improve with antibiotics. It was finally decided that the prosthesis should be extracted to remove the source of infection and the patient was operated on 13-01-2011 with extraction of both the cone and stem Figure 5.

A follow up CT showed major destruction of the hemipelvis. Figure 6 VAC (Vacuum Assisted Closure) was used to optimize drainage of the big wound hole and was shifted every 4 - 5 days for two weeks before the wound could be closed. The VAC treatment in combination with $\mathrm{Zyvoxid}^{\odot}$ (linezolid) $600 \mathrm{mg} 1 \times 2$ gradualy decreased the signs of infection and CRP started to go down. The patient was discharged on 03-02-2011 with a better general condition and wheelchair mobilization with tight follow up of liver enzymes, electrolyte and blood status from the health center.

She was admitted again 10 days after discharge because of Zyvoxid caused bone marrow suppression and increment of wound secretion. The antibiotic was changed and blood transfusion was given. Revision of the wound with use of VAC was done again. After a new series with VAC exchange the wound was clean and the secretion minimized. Final closure was done and the patient could

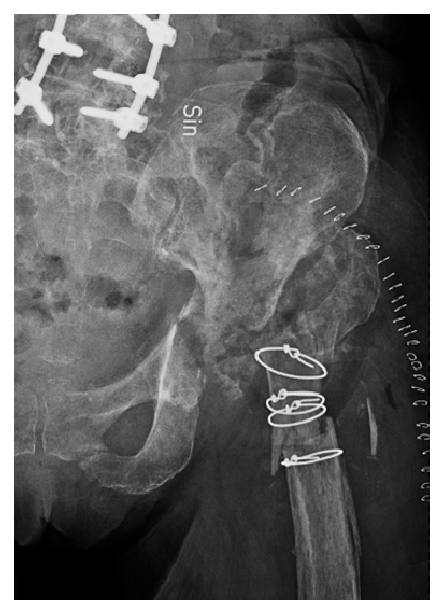

Figure 5. Complete removal of prosthesis due to infection and severe osteonecrosis of the pelvis.

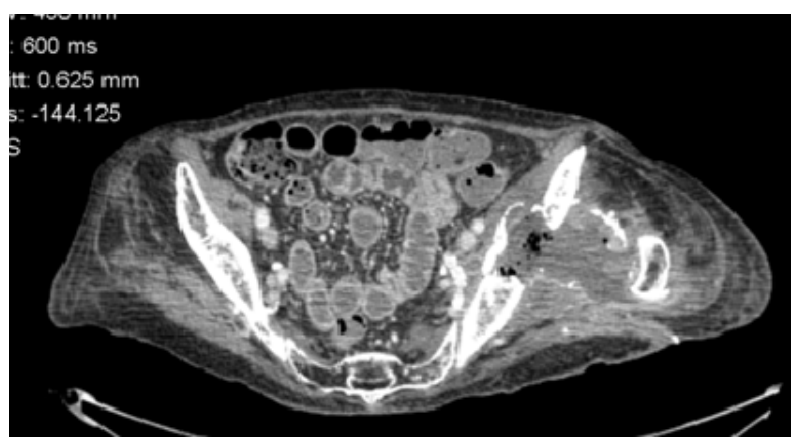

Figure 6. CT showing severe destruction of the left hemipelvis. 
leave the hospital in a good general condition. During follow up no new signs of active infection have been found. Because of the big osteonecrosis of the pelvis there is no plan for further reconstruction of the hip.

\section{Discussion}

Radiotherapy is given to various malignancies. Complications of irradiation may lead to severe functional impairment in patients who often have been cured of their cancer. Early and late infections as well as fibrosis, necrosis and ulceration can occur [1,2].

There are only few studies on radiation-induced osteonecrosis. In a retrospective study Massin and Duparac [3] described femoral neck fractures, osteonecrosis of femoral head and acetabulum after radiotherapy which were diagnosed years after the radiation treatment. Early changes of osteonecrosis include coarsening and disorganization of trabeculae and sclerosis; late changes include osteolysis, mechanical insufficiency, and subsequent fracture. These complications can prevent patients from returning to their preoperative functional level, despite successful tumor eradication [2].

In a retrospective evaluation using pelvic MR images of patients who underwent pelvic irradiation for uterine cervical cancer showed pelvic insufficiency fractures as frequent complications with 5-year cumulative prevalence of $45.2 \%$, and diagnosed at a median of 16.9 months after radiation therapy. The fracture sites were the sacrum body and alae, medial side of the iliac bone, the roof of the acetabulum, superior rami of the pubic bone, femoral heads, and L5 vertebra. Other complications associated with the radiation therapy, as determined by evaluation of the MR images, were osteolysis and avascular necrosis of the femoral head [4].

Post-irradiation lesions are often bilateral 21\%. They appear after a variable latency period of two to twenty years and they progress remorselessly [5]. Concerning our patient the pelvic destructions occurred 34 years after the radiotherapy and progressed fast.

It has been recommended that reinforcement rings are used for total hip replacement in irradiated hips [6]. In the present case it is obvious that even with this type of reinforcement early loosening might occur. And even a hemipelvic reconstruction prosthesis will get loose if the bone is heavily demaged. A Girdlestone procedure may be indicated and should always be considered in patients with known pelvic osteonecrosis due to irradiation.

In conclusion this case report shows that there might be problems with osteonecrosis more than 30 years after irradiation to the pelvis. This has to be considered when the different treatment options are discussed. In our case with the rapid destruction of the hip in a couple of months we should have considered the possibility of late severe osteonecrosis and examined the patient with CT or MRI. If we had done a resection osteotomy in the first place we would have speared the patient a lot of suffering.

\section{REFERENCES}

[1] R. Rudolph, T. S. Arganese and M. Woodward, "Ultra Structure and Etiology of Chronic Radiotherapy Damage in Human Skin,” Annals of Plastic Surgery, Vol. 9, No. 4, 1982, pp. 282-292. doi:10.1097/00000637-198210000-00003

[2] L. L. Pu and J. G. Thompson, "Salvage of the Exposed Irradiated Knee Joint with Free Tissue Transfer,” Annals of Plastic Surgery, Vol. 44, No. 3, 2000, pp. 334-339. doi:10.1097/00000637-200044030-00015

[3] P. Massin and J. Duparc, "Total Hip Replacement in Irradiated Hips. A Retrospective Study of 71 Cases,” Journal of Bone \& Joint Surgery, Vol. 77, No. 6, 1995, pp. 847852.

[4] R. J. Goitz, M. M. Tomaino, P. Smith, D. Hannallah and R. Sinha, "Treatment of Radiation-Induced Soft-Tissue Fibrosis and Concomitant Acetabular Osteonecrosis: A Case Report,” The American Journal of Orthopedics, Vol. 36, No. 4, 2007, pp. 56-58.

[5] J. W. Kwon, S. J. Huh, Y. C. Yoon, S. H. Choi, J. Y. Jung, D. Oh and B. K. Choe, "Pelvic Bone Complications after Radiation Therapy of Uterine Cervical Cancer: Evaluation with MRI,” American Journal of Roentgenology, Vol. 191, No. 4, 2008, pp. 987-994. doi:10.2214/AJR.07.3634

[6] J. Duparc and P. Massin, "Surgical Treatment of Radiation-Induced Lesions of the Hip in Adults," Bulletin de l'Academie Nationale de Medecine, Vol. 180, No. 8, 1996, pp. 1815-1836. 